\title{
El estudio del empresariado desde la perspectiva de la acción social
}

\author{
Esbozo de una propuesta para la historiografía del empresariado acuicultor
}

The study of entrepreneurship from the perspective of social action

\author{
Alexander Quintero B. \\ Universidad Surcolombiana, Colombia \\ alexanderquintero@mialegria.edu.co
}

\section{Introducción}

El estudio del empresariado en Colombia evidencia una creciente acogida por parte de la comunidad académica. Además del número de publicaciones y de investigadores, se cuenta un elevado número de cursos de historia empresarial ofrecidos en el pregradoy posgrados. Los balances historiográficos efectuados en el país concluyen que además de ser intuitiva la investigación en historia empresarial, existe la necesidad de identificar y establecer perspectivas teóricas que animen el debate académico.

El empresariado ha sido un tema ampliamente abordado desde las ciencias económicas. La sociología lo incluye en su agenda investigativa, aunque recientemente y con menor intensidad. Estos enfoques abordan la tarea investigativa con una perspectiva determinista, según la cual, son elementos estructurales los que determinan el surgimiento de un grupo de empresas. Más aún, la mayor parte de investigadores economistas se interesan en este tema como una manera de acercarse al fenómeno del crecimiento económico y no por el interés en el conocimiento del empresariado en sí.

Por lo tanto, la intención de esta reflexión es proponer una perspectiva teórica para el estudio del empresariado en Colombia, a partir de la propuesta construida por Pierre Bourdieu en torno a la teoría de la acción social. En particular, pretende que esta perspectiva teórica guíe la investigación de un caso en particular como lo es el fenómeno por el cual surgió un conjunto de organizaciones (empresas) que a su vez configuraron una nueva actividad económica en el departamento del Huila. El caso específico refiere a las empresas del sector acuícola que surgieron en el periodo de 1985 a 1995 y que hoy en día ubican a este Departamento en el liderazgo de la producción nacional.

El documento hará un breve recorrido por los balances de la historiografía colombiana. De allí, reseñará brevemente el caso propuesto para el estudio. Posteriormente, presentará los diversos enfoques empleados en el estudio de surgimiento de organizaciones (empresas). Finalmente, bosquejará una propuesta para la historiografía del empresario colombiano, construida desde la perspectiva de Pierre Bourdieu.

\section{La historia empresarial colombiana}

El estudio del empresariado colombiano es reciente dentro de la historiografía. Éste se remonta a unos primero trabajos en los años setenta para un aumento gradual a partir de los noventa. La situación fue algo cercana para el resto de Latinoamérica. Al respecto, Cerruti (2004) advierte que para el caso latinoamericano los estudios empresariales, que provenían desde la década de los años setenta, tomaron cierto auge desde la disciplina histórica a mediados de los ochenta como una rama de la historia socioeconómica que se confundía con la incipiente historia económica.

Además de que en el contexto colombiano se calificó a los estudios empresariales en los noventa como un campo en formación con abordajes desde perspectivas académicas 
diferentes a la historia oficial y conmemorativa, se advertía desde ese momento un "un largo camino por recorrer" para que se incorporaran los aportes de las ciencias sociales dentro de los recursos de investigación histórica. También se notó la existencia de un trabajo individual y aislado, con escasa y esporádica crítica académica, con una mínima vinculación a la comunidad académica internacional de la Business History (Dávila, 2003). Situación que no varió mucho en la primera década del siglo XXI. Así, los recientes programas de doctorados establecidos a mediados del año dos mil y la creación de la asociación de historiadores económicos se convirtieron en la esperanza para el desarrollo de una comunidad.

Una mirada reciente muestra que la mayor parte de las investigaciones del empresariado provienen de la historia económica y la sociología. Así mismo, se advierte una tendencia a investigar principalmente en torno a la historia regional y la formación del empresariado en las regiones. En menor proporción existen trabajos de la historia industrial y los sectores industriales, así como de empresas específicas, entidades bancarias, sectores económicos diferentes de los manufactureros, empresas de servicios públicos, asociaciones, biografías y autobiografías. En comparación con éstos, abunda la bibliografía sobre el tema de la industria enfocado desde la perspectiva de la política y la coyuntura económica, los que a pesar de su contribución a la economía no se consideran que hayan hecho aportes significativos a la historia empresarial (Dávila, 1996).

Uno de los problemas latentes en la historia del empresariado es que se encuentra limítrofe entre la historia, la sociología y la economía (Cohran, 1965) citado por Dávila (1996). Al respecto, señaló Casson (1982) citado por Dávila (1996): "Casi todas las ciencias sociales tienen una teoría del empresario, excepto la economía". En tal sentido, al interior de la comunidad académica colombiana empeñada en estas lides no se reconoce, aún hoy en día, marcos conceptuales que orienten con creces tanto el objeto de estudio como los debates académicos, por lo cual, se evidencia una aproximación más bien intuitiva. $\mathrm{Y}$ a pesar de la creciente acogida en el medio académico y el avance en cuanto a publicaciones, el estudio del empresariado, tal como lo afirmó Dávila (1986) se encuentra aún en relativo atraso en cuanto a términos teóricos. El mismo Dávila, ha planteado reiterativamente, como ejemplo, que las ideas de Schumpeter acerca de la innovación, la conducta económica como las de riesgo y oportunidades, el enfoque de Chandler, las ideas weberianas, entre otras, otorgarían mayor perspectiva a la historia empresarial (Dávila, 1986, 1996)

En resumen, la investigación en historia empresarial continua con la tendencia a ser de carácter artesanal y monográfico (Colmenares, 1990), citado por Dávila (2003), con una labor documental de diferentes grados de rigor historiográfico, sin apoyo, por lo general, en referentes teóricos explícitos, ni mayor interés por estudios comparativos o el esfuerzo de síntesis que permitan sustentar hipótesis para un elevado número de casos particulares de empresas o empresarios (Dávila, 1996).

\section{¿Cómo abordar la dinámica de creación de organizaciones en un espacio y tiempo específico?}

En atención a lo advertido hasta ahora, existe la imperiosa obligación de identificar perspectivas teóricas que permitan la aproximación y comprensión de la dinámica de creación de un conjunto de organizaciones y el papel que desempeñaron los agentes fundadores, tal como lo que se propone para las organizaciones del sector acuícola en el departamento del Huila.

Para continuar con éste propósito, haré una breve presentación del contexto en el cual se formó la actividad acuícola para desarrollar luego los posibles enfoques desde los cuales se puede abordar el proceso de investigación de tal realidad.

\section{Dinámica de la acuicultura en Colombia}

El inicio de la acuicultura en Colombia como actividad con incipiente formalidad se hace manifiesta a partir de la década de 1940. Se caracteriza este periodo por la introducción 
indiscriminada de especies que a la postre atentaron contra los ecosistemas locales. La primera etapa de éste proceso que abarca hasta la década de 1960 se caracterizó por la ausencia de infraestructura, equipos y desarrollo tecnológico. Es decir, la actividad nace como una alternativa de subsistencia para el sector agropecuario, específicamente campesino y como parte de una iniciativa para repoblar cuencas.

Con la creación del Instituto Nacional de RecursosNaturales-Inderena-en1968,laactividad acuícola experimentó otra dinámica. Proyectos desarrollados conjuntamente con instituciones internacionales como la Organización de las Naciones Unidas para la Alimentación -FAO-, la Asociación Internacional de Desarrollo -FAOy el Centro de Investigación para el Desarrollo -CID-, así como el gobierno de Taiwan, favorecieron el fomento de la actividad a través de la capacitación, transferencia de tecnología, asistencia técnica y estaciones piscícolas. A pesar de este impulso, la actividad acuícola continuaba como una actividad de fomento dirigida a pequeños productores al interior del país en departamentos como el Valle del Cauca y otros de la región Andina. (Beltrán y Villaneda, 2000). Se trató de un modelo agroacuícola fundamentado en la construcción de estanques de áreas no rentables y la entrega de alevinos gratuitos o subsidiados, para citar algunos. Una actividad de subsistencia sin excedentes para comercialización (Castillo, 2000).

En las décadas de 1980 y 1990 se desarrollaron proyectos importantes por parte de la Agencia Japonésa de Cooperación Internacional -JICAy el Centro Internacional de Investigación para el Desarrollo CIID (Canadá). Desde el estado también se orientó la política para el impulso de la actividad acuícola comercial con énfasis en camarón, trucha y peces ornamentales. En este contexto la actividad pasó de la subsistencia a la producción de excedentes para el comercio.

Corrido el año de 1985 nace en el Valle del Cauca la empresa Acuario Cali Ltda, primera organización dedicada a la investigación genética, interpretación y producción comercial de alevinos de tilapia roja y nilótica. Esta empresa se convirtió en 1987 en Acuicultivos de Cali Ltda. Por el año de 1988 se funda Colapia S.A, empresa que a través de Joint Venture accedió a la transferencia de paquetes tecnológicos de origen israelí y mercadeo internacional para la Tilapia Roja. La citada empresa inició operaciones con un espejo de agua de 64 hectáreas, lo que la convirtió en la empresa referente para Suramérica y proyectó a Colombia como el mayor productor en la región de Tilapia roja, con una producción cercana a las 4.000 toneladas al año. Las labores adelantadas desde estas organizaciones posicionaron al Valle del Cauca pionero en desarrollo técnico y tecnológico en la segunda mitad de los ochenta (Castillo, 2000).

De igual manera, en el año de 1984 fue creado en el departamento del Huila, específicamente en el municipio de Garzón, la primera experiencia significativa de cultivo de tilapia nilótica, más conocida como mojarra plateada. Esta empresa privada, de origen familiar, se instaló en la hacienda Castalia, sitio en el cual perdura y lleva su nombre hoy en día. De esta manera se tiene que al inicio de la década del 90 los departamentos del Valle del Cauca y el Huila se situaron como los pioneros en la producción organizada de Tilapia, que aunque modesto comparado con la producción actual, se establecían como los únicos en producir en forma organizada.

Con el incremento en el consumo per cápita de la Tilapia y la conformación de un mercado local, se inició en los noventa una década con buenos augurios para esta actividad. El proceso de apertura estimuló el crecimiento de la demanda lo que significó iniciar las exportaciones por parte de Colapia S.A hacia Estados Unidos. La actividad pasó de producir 3.040 toneladas en 1991 a 11.050 en el año de 1992. Esta dinámica hizo que el estado creara a inicios de la década un instituto especializado para la pesca y la acuicultura (INPA). En el ámbito internacional, se advertían similares iniciativas en países como Venezuela, Honduras, Costa Rica y Jamaica.

Con la asesoría de expertos internacionales, bajo el liderazgo del Colapia S.A, inicia la utilización de "residuos" del proceso como cabezas, espinazos, recortes y la piel de la tilapia. 
En 1993 fue fundada en el Valle del Cauca la empresa Camarpez destinada a la curtiembre de la piel de la tilapia. La producción se afianzó al punto que para el año de 1995 se produjo 16 mil toneladas de tilapia, doblando la producción de camarón y triplicando la de trucha y cachama (Inpa, 1997).

El crecimiento de la demanda interna del producto y el cierre de la principal empresa Colapia S.A en 1997, estimuló la puesta en marcha en proyectos de cultivo de tilapia bajo la modalidad de jaulas flotantes en los embalses de los departamentos del Huila, Cauca y Tolima. De esta manera, la producción de tilapia se aproximó a las 20 mil toneladas en 1999, superando esta cifra a partir del año 2000 (Castillo, 2003).

Precisamente, será la década del año 2000 en la que se intensificará el cultivo de la tilapia en jaulonesy conelusodeabundantescuerposdeagua existentes en los embalses. El departamento del Huila se posicionará como líder de la producción nacional de tilapia, logrando una producción cercana a 16 mil toneladas, equivalentes al 30\% de la producción nacional (Gobernación del Huila, 2013), destinadas mayormente al consumo del mercado local, con exportaciones menores al mercado norteamericano.

De acuerdo con un informe de la gobernación del departamento (Gobernación del Huila, 2013) el Huila se consolidó en la década del año dos mil primer productor nacional de tilapia aportando el $53 \%$ de la producción total, lo que generó alrededor de 5.288 empleos directos con ingresos por exportaciones anuales de 5.5 millones de dólares. En la actualidad coexisten tres tipos de productores: 1) pequeños productores que generan cerca del $20 \%$ de la producción cuyo mercado es el intermediario; 2) los medianos productores, que producen por encima de 80 toneladas por año y aportan el $25 \%$ de la producción y 3) los grandes productores con volúmenes de producción superior a las 800 toneladas por año, produciendo alrededor del $60 \%$ del total de tilapia. Este grupo está integrado por 9 empresas que integran toda la cadena productiva, es decir, producen desde el alevino hasta el proceso industrial del filete.
Tal como se advierte, la dinámica apreciada en el departamento del Huila en cuanto a la creación de organizaciones empresariales formales, insertas en la lógica del mercado acuícola es reciente. La primera de ellas, data de 1984. Sin embargo, fue en la década de los noventa que inició la dinámica conocida hoy en día. Cinco de las nueve empresas calificadas como gran productor fueron establecidas en la citada década. Dada la importancia que registran estas organizaciones en el contexto local y nacional, así como la particularidad del fenómeno y su manifestación reciente sin ningún referente en la localidad, se hace atractivo comprender tal manifestación.

Enfoques para abordar el estudio de la dinámica de formación de organizaciones

En este apartado se hará un despliegue de las perspectivas teóricas que han estudiado la formación de organizaciones empresariales. Se incluyen, igualmente, perspectivas, que si bien no han abordado el estudio de los empresarios o la acción empresarial en procura de la creación de tales organizaciones, si brindan elementos para la comprensión de esta situación.

Para empezar, se advierte que el estudio de la dinámica de formación de un conjunto de empresas en un espacio y tiempo específicos es de vieja data. Ha sido un tema de marcado interés propuesto inicial y continuamente desde las ciencias económicas en procura de dar respuesta al estancamiento o el desarrollo productivo de una región. Por otra parte, la inquietud en cuanto a la formación de un conglomerado de organizaciones ha sido tímidamente tratada desde la sociología y los recientes estudios organizacionales.

Desde la perspectiva económica, la aparición y formación de un conjunto de organizaciones en el marco de una actividad específica es la consecuencia de las políticas macroeconómicas que desbordan los propósitos de los actores y de una comunidad local o regional. También al hecho de superar las limitaciones y obstáculos estrictamente locales como la baja capacidad empresarial, los problemas de financiamiento $y$ 
las deficiencias de recursos humanos (Gómez, 2005). De acuerdo con Smitchz (1995) en las décadas de 1950 y 1960 se atribuyó a la protección y sustitución de importaciones la creación de nuevas organizaciones productivas. Inclusive, otra línea de pensamiento condicionó este fenómeno a la presencia de factores culturales que promovían o frenaban el surgimiento de individuos con un conjunto de habilidades para el riesgo empresarial.

Por otra parte, los conglomerados o la formación de un conjunto de organizaciones en el ámbito de una actividad productiva, asociados al crecimiento económico, fue expuesto inicialmente dentro de la teoría clásica por Marshall en 1920: "la aglomeración de firmas involucradas en actividades similares o relacionadas genera un conjunto de economías externas localizadas que reducen los costos para los productores que forman parte". Al respecto, Krugman, citado (Smitchz, 1995) agregó tres razones o fuentes de economía externa que incentivan a las firmas a ubicarse geográficamente: 1) la concentración de obra de mano especializada 2) el mayor acceso a insumos intermedios y 3) los aprovechamientos tecnológicos Así mismo, se debe considerar que dentro de esta perspectiva los factores históricos y naturales juegan un rol importante en el establecimiento y localización de las organizaciones. No obstante, el sostenimiento, desarrollo y por tanto la perdurabilidad de las mismas estará condicionado por otros factores como la capacidad de innovar y de mejorar, la especialización y el mejoramiento del capital humano, para citar algunos (Gómez, 2005).

\section{- La perspectiva del Distrito Industrial}

El Distrito Industrial es un concepto propio del campo de la economía política que con variantes interpretativas se ha difundido a otros campos disciplinarios. Según Becattini (1988) el concepto de Distrito Industrial es propio de Alfred Marshall, quien previó las ventajas de las economías externas que presentaba el agrupamiento de pequeñas y medianas empresas subdivididas en fases productivas, ubicadas en un área geográfica específica. Así, la agrupación es el resultado de la cercanía e interacción de distintos agentes en procesos propios de su actividad económica, situación que definió Marshall como ambiente industrial, la cual designa el entorno socioeconómico de donde emergen beneficios provenientes de procesos deimitación, aprendizaje, innovación y adaptación tecnológica. Tales procesos se concretan a partir de la cooperación de los agentes (Piña y Morales, 2009).

El mismo Marshall indicó, años después, que la población de empresas que integran la industria se "interpenetra" con los habitantes del mismo territorio, los que a su vez comportan un conjunto de características socio-culturales adecuadas para un proceso de desarrollo de pequeña empresa. Lo cual indica, por una parte, que la historia y la geografía cuentan en la conformación del distrito, y por otra, que el Distrito Industrial demanda una simbiosis especial entre la actividad productiva y la vida comunitaria. El proceso productivo llevado a cabo dentro del distrito debe permitir la descomposición en fases y la posibilidad de transportar en el espacio y el tiempo los productos entre las fases. Esta situación es la que permite la creación de una red en el mercado local y la subdivisión del trabajo que integra a los miembros de la comunidad en múltiples posiciones (Becattini, 1988). Entonces a partir de la idea de ambiente industrial, Becattini asocia la noción de Distrito Industrial en tanto reconoce una identificación de las comunidades de los distritos con los sistemas productivos.

La teoría de los Distritos Industriales, posterior a Marshall, comportó avances teóricos en Italia a partir de la década de los años setenta. Un nuevo modelo de sistema productivo fue incubado en este país en un espacio no previsto después de la segunda guerra mundial. La particularidad de este nuevo sistema productivo, en comparación con tradicionales zonas industriales, fue el tamaño de la empresa y las complejas relaciones con la comunidad local. Dos enfoques se advierten en las definiciones teóricas de los Distritos Industriales italianos: uno que hace hincapié en dimensión económica de la red de empresas que componen el distrito, es decir la estructura de las relaciones entre ellos, y otro, que involucra elementos socioeconómicos que incrusta a las empresas en el sistema social de la localidad. 
Bagnasco (1977) citado por Becattini (1988), define el Distrito Industrial como un sistema productivo geográficamente localizado, basado en la intensa división local de actividades entre pequeñas empresas especializadas en los diferentes procesos de producción y de la distribución de un sector industrial o una actividad dominante, existiendo múltiples relaciones entre la empresa y la comunidad local, tanto dentro como fuera del mercado, relaciones basadas en la confianza y la reciprocidad.

Siguiendo a Becattini (1988) el Distrito Industrial se puede considerar como:

Un gran complejo productivo en el que la coordinación entre las distintas fases y el control de su funcionamiento regular, no se lleva a cabo mediante unas reglas preestablecidas $\mathrm{y} / \mathrm{o}$ mediante mecanismos jerarquizados, sino mediante la confianza en la combinación del juego mecanizado del mercado con un sistema de sanciones sociales impuestas por la comunidad. La proximidad permite a las empresas gozar de una economía de gran escala...esta explicación no mantiene que la pequeña empresa pueda ser tanto o más eficaz que la grande, sino que, si se dan las condiciones anteriormente expuestas, una población localizada de pequeñas empresas puede alcanzar, en la producción fraccionada $\mathrm{y}$ variable, niveles de eficacia al de las grandes empresas...(p. 7)

Cabe precisar que de acuerdo con Becattini (1988)noes precisoincluir dentro deesta categoría a un grupo de empresas pequeñas establecidas en un área y bajo el dominio de una más grande. Tampoco se incluyen las áreas urbanas en las que intervienen pequeñas empresas en una misma fase del sistema productivo, tal como los barrios industriales. En suma, para Becattini (1998) el Distrito Industrial es ante todo una entidad socioterritorial, caracterizada por la presencia activa de una comunidad de personas y un conjunto de empresas, ubicados juntos en una zona natural e históricamente determinada en la cual la comunidad y las empresas tienden a fundirse.

Para Humprhrey (1995) la característica fundamental de un Distrito Industrial es su organización. Es decir, que el éxito no se logra por el acceso ventajoso a bajo costo de los factores de producción, sino más bien a la eficaz organización del aspecto social y económico, en la interacción de la localidad con la pequeña empresa. La organización puede variar, pero el elemento clave es la existencia de redes fuertes a través de la especialización y la subcontratación entre las empresas mismas. Este mismo autor citando a Piore y Sahel (1984), quienes concretaron la experiencia de lo que se denominó el modelo de la Tercera Italia, argumenta que la vitalidad del distrito está en las comunidades de las empresas agrupadas, que se basa en la competencia cooperativa entre las empresas y las amplias capacidades de la comunidad trabajadora; lo que permite la disposición para la innovación y la elaboración de una amplia gama de productos en pequeñas cantidades.

De todo ello se tiene que un modelo de Distrito Industrial involucra al menos: a) un territorio delimitado, b) una comunidad de personas, c) un conjunto de pequeñas y medianas empresas especializadas, d) división y especialización de la obra de mano, e) actores institucionales, f) competencia y cooperación, g) diversificación e innovación en la producción.

\section{- La perspectiva del Clúster}

De acuerdo con Gómez (2005) pueden encontrarse numerosos estudios sobre los clúster provenientes de diferentes corrientes teóricas, lo cual ha llevado a una confusión sobre lo que son. Desde la perspectiva teórica promulgada por Porter (1998), el clúster hace referencia a la concentración geográfica de empresas e instituciones interconectadas en una actividad industrial particular. Incluye proveedores, fabricantes de productos complementarios, entidades gubernamentales, universidades, organismos para la fijación de normas, grupos de reflexión, asociaciones comerciales, de investigación, apoyo técnico y hasta canales y clientes. El clúster representa una especie de organización espacial de los mercados. Es una forma alternativa de organización de lo que Porter denomina cadena de valor. La proximidad de la empresa y las instituciones en un solo lugar, 
así como los repetidos intercambios entre ellas fomenta una mejor coordinación y confianza.

Los clúster pueden ser vistos igualmente como procesos si son observados como una masa crítica de firmas interdependientes conectadas geográficamente (Rosenfeld, 2002). No se trata solo de un flujo físico de bienes y servicios, sino también constante intercambio de información, conocimiento y tecnología que adquiere forma con el tiempo. Es decir, éste es un modo de entender la manera como la economía funciona y organiza sus estrategias para el logro de resultados. Desde esta perspectiva, el clúster al promover la especialización, el funcionamiento de redes, las relaciones horizontales y verticales y lograr economías externas y la eficiencia colectiva; construye ventajas competitivas. Este ambiente es promotor igualmente para el surgimiento de nuevas formas de organización.

De acuerdo con López y Calderón (2006) en el concepto de clúster ha prevalecido una mirada económica, tanto en la perspectiva teórica enunciada por Porter como en los trabajos empíricos. Es decir, el clúster se focaliza en las economías de escala y eficiencia colectiva a través de los procesos de complementariedad entre las organizaciones, de tal forma que generan ventajas competitivas para el conglomerado como un todo. Entonces, la atención se centra en la robustez de los eslabones empresariales en cuanto a capacidades, así como en la calidad e intensidad de las relaciones económicas e institucionales entre los agentes del conglomerado, antes que establecer el proceso que dio origen a un conglomerado de empresas que luego integraran el clúster.

- La perspectiva del Institucionalismo

En las últimas décadas del siglo pasado resurgió el interés por el estudio de las Instituciones en las ciencias sociales. Esto, como rechazo a la teoría neoclásica de la economía y al viejo institucionalismo, sobre la base que tanto los arreglos institucionales como los procesos sociales son fundamentales para comprender la sociedad. Los postulados neoinstitucionalistas permiten abordar ciertos fenómenos organizacionales.
Sin embargo, aproximarse a la formación de un conglomerado de organizaciones desde la perspectiva neoinstitucionalista demanda superar el conflicto generado en el concepto mismo de institución, el cual varía según el enfoque asumido, sea económico, sociológico o político.

De acuerdo con Dimaggio y Powell (1999), el institucionalismo tiene arraigo en la economía política de Veblen y Commons y la sociología de Parsons y Selznick. En los primeros, por cuanto consideraron los mecanismos generadores de la acción social y económica. En cuanto a la sociología, puesto que se intentaba comprender las interconexiones entre la organización, la economía y la sociedad. También se trataba de una oposición a los principios de la economía clásica y su noción de racionalidad ilimitada e inclinación utilitarista de parte de los agentes económicos. De allí, el Institucionalismo pasó a ser parte de la Nueva Economía Institucional por cuanto las transacciones -unidad primaria de análisis- originan clases específicas de instituciones económicas. En esta perspectiva, las instituciones imponen restricciones al comportamiento individual, limitando las estrategias adoptadas por los actores para el logro de los objetivos.

El Neoinstitucionalismo nacerá a finales de la década del setenta, también como una oposición al enfoque racional y la visión utilitarista del comportamiento humano postulado desde la teoría neoclásica, y en particular como una afronta a los costos de transacción y los derechos de propiedad. El Neoinstitucionalismo, a partir de la teoría de la acción práctica, se ocupará de la actuación humana y de las instituciones, las organizaciones y el entorno. La naturaleza y el comportamiento humano será considerado como no predecible, en tanto que los intereses estarán determinados por las instituciones (Dimaggio y Powell, 1999)

Por lo anterior, una mirada amplia al nuevo institucionalismo, desde la perspectiva sociológica, supone la existencia de un vínculo entre las acciones humanas tanto individuales como grupales y las instituciones. Las instituciones influyen en las acciones y ellas mismas son el resultado de dichas 
acciones (Scapens, 1994). Los agentes individuales como los grupos procuran sus respectivos propósitos en un contexto colectivamente restringido, es decir, en un contexto de instituciones, entendidas éstas como patrones organizados de normas y roles socialmente construidos así como conductas socialmente prescritas que se esperan de quienes desempeñan tales roles, los cuales se crean y recrean en el tiempo (Goodin, 2003).

Desde el Neoinstitucionalismo sociológico se podría interpretar el surgimiento de un conjunto de organizaciones a partir del ambiente institucional. En tal sentido, Meyer y Rowan (1977) plantean que la noción fundamental dentro del nuevo institucionalismo sociológico son las reglas institucionales y la manera como afectan las estructuras y el desempeño de éstas. Definen este ambiente como la aprobación de las reglas del juego, constreñimientos y obligaciones aceptadas por los agentes. A su vez, estos agentes asumen formas de comportamiento que son aprobadas legítimamente por el medio, e interiorizadas como actos posibles que se delimitan a través de sistemas compartidos de prácticas que se vuelven rutinas homogenizantes. Scott (2008) concuerda al afirmar que al entorno puede corresponderle el papel de elaborar reglas y requerimientos que los individuos, grupos y organizaciones deben acoger si desean obtener soporte y legitimidad.

Según Jepperson (1991) una institución es un orden o patrón social que ha alcanzado un cierto estado o propiedad. De acuerdo con Dimaggio y Powell (1999) las instituciones son las reglas de juego de una sociedad. En este mismo sentido, las organizaciones se integran y conforman lo que estos autores califican como un campo organizacional. Es decir, grupos de organizaciones que interactúan directa o indirectamente y que, tomadas en conjunto, constituyen un área reconocida de vida institucional.

El campo como concepto teórico para la comprensión de los fenómenos administrativos y organizacionales proviene de la sociología, concepto desarrollado, entre otros, por Bordieu. La noción de campo organizacional ajustada al estudio de las organizaciones es referido igualmente a aquel espacio social en el que se materializan las relaciones con grupos similares y complementarios, que se ven influidos por presiones institucionales que tienden a homogenizar los comportamientos de los agentes que integra la comunidad (Dimaggio y Powell, 1999). De aquí se desprende que todo campo tiene un elemento histórico, relacional, relativo y complejo. los campos existen en la medida que están institucionalmente definidos, por lo que las interacciones llevadas a cabo por las organizaciones y las instituciones determinan la estructuración. La existencia de las organizaciones como productos de la acción humana se circunscriben al desarrollo institucional, el cual a la postre posibilita o no su creación.

Al margen del nuevo institucionalismo sociológico (NIS) expuesto anteriormente, se promulgó el nuevo institucionalismo político (NIP), el cual presenta un nivel análisis en el ámbito organizacional, específicamente intenta establecer los mecanismos que regulan el comportamiento de los individuos en un ámbito, el cual inicialmente es la organización. Bajo esta perspectiva, igual que en el NIS, la regla es lo fundamental y ésta es construida socialmente. De esta manera, la acción de los sujetos se institucionaliza cuando las reglas han sido institucionalizadas, y esto se lleva a cabo a través de la rutinización de las prácticas (otro concepto desarrollado igualmente por Bordieu). Así, las instituciones políticas se entenderán como un conjunto de reglas y rutinas relacionadas entre sí, que definen adecuadamente las acciones en términos de las relaciones entre las funciones y las situaciones (March y Olsen, 1997)

En el planteamiento de March y Olsen son fundamentales la interpretación y la construcción del significado, juntas juegan un papel preponderante en la institucionalización de la acción y las reglas. Es decir, en el NIP la institución ofrece una pluralidad de reglas, de las cuales el individuo interpreta y otorga sentido a la(s) mas adecuada(s) para una situación dada, esto lo hace, no en función de sus intereses individuales, sino en función de su rol o identidad que lo constriñe a actuar en 
consonancia con ellos. La cuestión aquí es que el rol y la identidad están socialmente determinado (Scott, 2008). Este planteamiento es totalmente contrario a los supuestos de racionalidad, en la que los individuos realizan una escogencia a partir de las preferencias, las alternativas y las consecuencias. En el NIP las reglas bajo las cuales los individuos deciden actuar están fijadas por lo marcos institucionales interiorizados, vale decir, la experiencia social, cultural y política, para citar algunas, las cuales entran a incidir.

- La perspectiva de la Estrategia como práctica

Tradicionalmente se acepta que la estrategia es un atributo de las empresas, algo que las organizaciones tienen. Pues bien, contrariamente a esta tradición ha germinado un movimiento que se ocupa en el hacer de la estrategia, conocido con el nombre de "estrategia como práctica", distinguida con la abreviatura "s-as-p". La estrategia es también una actividad llevada a cabo por las personas, algo que la gente hace y no es simplemente una propiedad de la organización. En este enfoque la estrategia se concibe como un resultado de una actividad social, algo que pasa por las acciones, interacciones $y$ negociaciones de múltiples actores, que se basan en prácticas institucionalizadas (Whittington, 2006). Nuevamente se recurre al "giro" dado en las ciencias sociales hacia las prácticas sociales, concepto desarrollado fundamentalmente por Bordieu. A juicio de Chia y MacKay (2007) la investigación se volcó hacia las prácticas "microsociales" de la organización.

Desde el punto de vista de las prácticas sociales, la estrategia es derivada de las prácticas cotidianas, es decir de patrones y disposiciones interiorizados en los individuos. En tal sentido, la estrategia no es resultado de iniciativas intencionales y deliberadas producto dela fijación de metas (Chia y MacKay, 2007). De acuerdo con los anteriores autores, se tiene que los trabajos de Pettigrew y Johnson en la década de 1980 (El gigante despierta y Cambio estratégico y El proceso administrativo), conformaron la base de lo que más tarde se denominaría la estrategia como práctica. Posteriormente, en la década del año en comento, se profundizaría en este "paradigma" con los aportes de Jarzabkowski y Whittington.

Bosquejo de una propuesta para la historiografía del empresariado colombiano

Tal como se apreció en la exposición que antecede a las presentes líneas, el estudio de las organizaciones empresariales evolucionó desde elenfoquedelaeconomíahastalaincorporaciónde diversas disciplinas para su análisis. Inicialmente se construyó una respuesta desde las economías externas y la localización hasta la inclusión de la teoría institucional. Bajo la denominación de conglomerados, distritos industriales y cluster, se identificaron elementos distintivos tales como la proximidad geográfica, los múltiples actores, el tamaño de las organizaciones, las actividades especializadas, el entorno institucional, la cooperación y la competencia, para citar algunos, que por una parte, explican el origen de un grupo de organizaciones y por otra dan cuenta de la manera como las citadas organizaciones sobreviven y se desarrollan.

La perspectiva económica deja ver que cada conglomerado, distrito o cluster fue único en su proceso de conformación y permanencia. También, que no existe un orden estricto de incidencia de los elementos señalados como distintivos. Las manifestaciones empresariales fueron relativas y singulares. Ahora, cabe preguntarse si las consideraciones advertidas desde el enfoque de la economía responde a las siguientes inquietudes: ¿son suficientes las economías externas, red de empresas interconectadas, el sistema social de una localidad, la especialización y la complementariedad y las instituciones, para comprender la manera como aparecen y se conforman un conjunto de organizaciones empresariales en un espacio y tiempo específico?.

La pretensión del presente escrito conduce a sostener que no son suficientes las citadas explicaciones. No tiene sentido abordar la comprensión de realizaciones humanas sin seres humanos. Una organización o un conjunto de éstas no tiene una existencia autónoma, ni mucho menos independiente de los individuos. La creación o puesta en marcha de una organización 
empresarial es una acto, una respuesta o una construcción de sentido de la realidad; efectuada por unos sujetos que a la postre conforman una clase particular. No todos los sujetos crean organizaciones empresariales. Tampocotodoslos sujetos cuentan con los medios requeridos. Cobra sentido abordar a estos sujetos y las condiciones bajo las cuales crean las organizaciones. Tiene sentido estudiar a los actores, sus papeles, el escenario y la manera como se producen a sí mismos y a otras realidades. La acción de los hombres es la fuerza motriz para toda creación, razón por la cual se sostiene su inclusión.

Una teoría de la práctica: elementos de Bourdieu para la comprensión de la práctica empresarial.

- De las estructuras sociales y el objetivismo a la acción social y el subjetivismo

En el texto "Estrategias de la reproducción y modos de dominación", Bourdieu (2011) se cuestiona: ¿por qué y cómo el mundo social dura, persevera en el ser, se perpetúa?. El mundo social, en la tradición sociológica, ha tenido un tratamiento objetivista. Es decir, relaciones objetivas estructuran la acción y las representaciones que se hacen los individuos. Bourdieu propone una inversión a la relación estructura-acción instalándose en la forma de conocimiento denominado praxeológico. Éste, tiene por objeto no solo el sistema de relaciones objetivas sino las relaciones dialécticas entre esas estructuras objetivas y las disposiciones estructuradas en las cuales ellas se actualizan y tienden a reproducirlas. Se trata de una inversión de la problemática, puesto que si el mundo social objetivo, con un sistema de relaciones objetivas e independientes de las conciencias y de las voluntades individuales, funda las condiciones de posibilidad, la praxeología, que no anula la relación entre las estructuras y prácticas o representaciones, amplía o integra las interacciones simbólicas y la representación que los agentes pueden hacerse, por anticipación o por experiencia de los otros agentes confrontados. En términos de Bourdieu, las relaciones sociales son lo que son y lo que se hace (Bourdieu, 2012).
Parodiando a Bourdieu se tiene que el sentido de lo social es irreductible a "la voluntad del artista" tanto como a la "voluntad de la época" y a las experiencias vividas que la "obra suscita en el espectador". Lo social se constituye constituyendo un sistema de relaciones irreductibles tanto en cuanto a las prácticas en las que ella se realiza y se manifiesta, cuanto a las intenciones de los sujetos y a la conciencia que ellos pueden tomar de sus obligaciones y de su lógica (Bourdieu, 2012, 189). En las interacciones de una coyuntura particular las posiciones objetivas de los agentes en la estructura social igualmente han de ser consideradas.

Una clara distancia del Estructuralismo y del Institucionalismo se aprecia en Bourdieu, quien siguiendo a Leach, acoge: "yo postulo que los sistemas estructurales en los cuales todas las vías de acción están estrechamente institucionalizadas son imposibles. En todo sistema viable, debe existir un espacio donde el individuo es libre de hacer elecciones que le permitan dar vuelta al sistema en su beneficio". El estructuralismo es impotente para integrar en la teoría todo lo que se desprende de la ejecución. $\mathrm{Al}$ dejar quieta la producción de regularidades, el objetivismo trata a los objetos construidos, por ejemplo cultura y clase social, como realidades autónomas dotadas de una eficiencia social, capaz de obligar a la práctica (Bourdieu, 2012, 193-195).

Para escapar del realismo de la estructura, como una totalidad construida por fuera de la historia del individuo y del grupo, Bourdieu (2012) plantea que se debe ir del opus operantum al modus operandi -de la obra hecha al modo de operar- al principio de producción de lo observado y construir la teoría de la práctica, más exactamente, del modo de generación de las prácticas, una dialéctica de la interiorización de la exterioridad y la exteriorización de la interioridad.

Acometer al empresariado colombiano desde la propuesta de Bourdieu demanda hacer uso de una red de conceptos propuestos por el mismo autor, los cuales se desarrollan a continuación: 
- El espacio social

En general, los conceptos en Bourdieu, son conceptos construidos en el sentido lógico. Es decir, tienen la existencia propia de las teorías, en tanto es el producto de una clasificación que permite explicar y prever las cosas clasificadas. (Gutiérrez, 2011).

En tal sentido, se advierte que Bourdieu pasó paulatinamente de la consideración de clase social a la de espacio social (Baranger, 2004, citado por Gutiérrez, 2011). El concepto de clase social, que deja de ser una sustancia para pasar a ser una relación, es referido a:

Conjuntodeagentesqueseencuentransituados en unas condiciones de existencia homogéneas, que imponen unos condicionamientos homogéneos y producen unos sistemas de disposiciones homogéneas, apropiadas para engendrar unas prácticas semejantes, y que poseen un conjunto de propiedades comunes, propiedades objetivadas, a veces garantizadas jurídicamente (como la posesión de bienes o poderes) o incorporadas, como los habitus de clase...la clase construida se define como el conjunto de todos los factores que operan en todos los dominios de la práctica: volumen y estructura del capital definidos puntualmente y en su evolución(Bourdieu, 1988, 100).

La clase social se construye fundamentalmente en la distinción entre dos aspectos de la situación de clase: la condición y la posición. La condición de clase esta ligada a cierto tipo de condiciones materiales de existencia y de práctica profesional, en tanto que la posición de clase se refiere al lugar ocupado en la estructura de las clases respecto a las demás. Una clase social está ligada a sus relaciones objetivas con las demás clases y a la vez posee propiedades ligadas a las relaciones simbólicas que sostiene con sus miembros entre sí y con las demás clases. Siendo así, la condición de clase se define a partir de categorías de posesión y desposesión de bienes o del manejo de los mismos, en tanto que las relaciones simbólicas son maneras de usar y de consumir bienes, asociados a los estilos de vida. Una clase es definida por la estructura de las relaciones existentes entre todas las propiedades pertinentes y los efectos que ejercen sobre las prácticas (Gutiérrez, 2011).
Sin embargo, en Bourdieu, primero está el espacio social y luego aparecen las clases. Así el espacio social se construye a partir del volumen y estructura del capital, así como la evolución histórica (trayectoria). El volumen del capital, o sea, el conjunto de recursos y poderes efectivamente utilizables, conformado por el capital económico, cultural, social y simbólico, determina las diferencias primarias y con ello las condiciones de existencia. La estructura del capital, o sea la forma en que está distribuido ese capital entre las diferentes especies, determina diferencias secundarias y con ello las distintas fracciones de clase. Esto implica que en la reproducción de las clases entran en juego tanto las condiciones materiales como las simbólicas (Bourdieu, 2011).

El capital en Bourdieu se entiende como recursos que ponen en juego los agentes para obtener ventajas en los diferentes campos. El capital es a su vez un producto del mismo campo y existe únicamente en relación con éste. No se refiere exclusivamente a el capital económico, acumulable en bienes e infraestructura de valor monetario, sino que involucra la acumulación de honor, honradez, solvencia, competencia, pundonor, elementos aglutinados en el capital simbólico. También el capital cultural que puede ser incorporado en el seno de la familia o de una circunstancia en particular, el objetivado que es visible y se materializa en la acumulación de objetos extraordinarios y el institucional reflejado en los títulos y diplomas. Finalmente se encuentra el capital social definido por la red de relaciones que establece el agente en su campo (Gutiérrez, 2011). La producción o la posesión de un determinado tipo de capital determina el dominio en las relaciones sociales. En el juego, los agentes transforman estos capitales entre si, de allí que el concepto de campo funcione también como un mercado de bienes materiales o simbólicos.

\section{- El Campo}

El campo es el concepto que engloba a los restantes en la teoría de Bourdieu. Un campo es básicamente un espacio social de acción, de influencia. En términos analógicos es como un juego. Se establece una red de relaciones 
atravesadas por conflictos, pujas o lucha de intereses entre los agentes. En Bourdieu, los seres humanos son referidos como agentes en tanto tienen capacidad de agencia, es decir, no son sujetos estáticos sino que tienen capacidad de maniobra. Precisamente, los agentes luchan por un capital simbólico y material, y en este juego, procuran acrecentar y reproducir su capital con alianzas, intercambios, participación en instancias, para citar algunas. El conjunto estructurado de campos conforma la estructura social, la cual se dinamiza con la misma dinámica proporcionada por la acción de los agentes en el campo (Bourdieu, 2012).

Al interior del campo se encuentran los agentes con su capital. El capital es un propiedad que se carga, es llevado consigo por cada agente. Los campos son definidos por la relación de fuerza que el capital ejerce y por las acciones de los agentes para conservar y acrecentar su capital.

\section{- El Habitus}

\begin{abstract}
“Cada agente, lo sepa él o no, lo quiera o no, es productor y reproductor de sentido objetivo: porque sus acciones y sus obras son el producto de un modus operandi del que él no es el productor y del cual no tiene el dominio consciente, ellas encierran una intención objetiva...que exceden siempre sus intenciones conscientes" (Bourdieu, 2012, 214)
\end{abstract}

Entonces un habitus se entiende como un sistema de disposiciones durables y transportables que, integrando todas las experiencias pasadas, funciona en cada momento como una matriz de percepciones, de operaciones y de acciones (Bourdieu, 2012, 205). Las disposiciones, asumidas como estructuras mentales que guían las acciones en el contexto del agente conforman los habitus. Las disposiciones son adquiridas en la socialización, vale decir, en la experiencia previa de los agentes. Las disposiciones conforman el sistema subjetivo de expectativas y predisposiciones de los agentes. De esta manera, el habitus constituye el principio de toda acción, es el productor de las prácticas.

El habitus se asemeja a la regla, lo cual transmite la sensación de que el agente, al estar en un campo particular, se comporta de una manera específica acorde al habitus. La cuestión aquí es que no se trata de una determinación objetiva del comportamiento del agente, sino de unos condicionamientos, ya que existiendo reglas dentro del campo cada agente es capaz de decidir y adecuar por tanto su propio movimiento. Esta situación se comprende mejor en lo que Bourdieu (2012) denomina dialéctica de interiorización de la exterioridad y exteriorización de la interioridad. El hábitus, como estructura objetiva, producto de la experiencia, produce practicas, tales practicas producen regularidades ajustadas a las exigencias de la situación directamente afrontada. Por tanto, las practicas están atadas al habitus y a las condiciones de ejecución de ese habitus.

En cada uno de nosotros...hay un hombre de ayer...es predominante en nosotros, porque el presente es muy poca cosa comparado con ese largo pasado...solo que a ese hombre del pasado no lo sentimos, porque el es inveterado en nosotros, constituye la parte inconsciente de nosotros mismos...por el contrario, de las adquisiciones más recientes...tenemos un vivo sentimiento, porque en tanto que recientes, ellas no han tenido el tiempo de organizarse en el inconsciente...lo aprendido...aparece como el sentido objetivo...habitus objetivamente concertados que producen prácticas y obras, ellas misma objetivamente concertadas (Bourdieu, 2012, 207).

- Las prácticas y las estrategias de reproducción

La práctica es más que un simple comportamiento tomado en su valor facial. No es tampoco una relación mecánica determinada por las condiciones previas, modelos, normas o roles. La práctica siendo necesaria y relativamente autónoma es el producto de la relación dialéctica entre una situación y un habitus (Bourdieu, 2012). Las prácticas son producidas dentro de un espacio social (campo), es decir se relacionan con las prácticas constitutivas de los demás, creando modos de reproducción social diferenciados. Las prácticas son las acciones que los agentes acometen en procura de sus objetivos.

En su teoría de la acción social Bourdieu pasa de la "regla a las estrategias". La estrategia la identifica con la noción de práctica. Trata de rescatar al 
agente quien oficia como productor y es resultado simultáneo y dialéctico de las condiciones objetivas de vida, externas e incorporadas (Gutiérrez, 2011). Los agentes poseen capital y sus prácticas son estrategias de reproducción y transformación de ese capital. Como agentes poseedores de capital solo pueden mantener su posición en la estructura social a través de la reconversión del capital. Entonces en palabras de Bourdieu (2011, 118) las estrategias de reproducción son "secuencias objetivamente ordenadas y orientadas de prácticas que todo grupo debe reproducir para reproducirse como grupo"

\section{Reflexiones finales}

Los balances de la historiografía en Colombia evidencian que el estudio del empresariado comporta en el país una acogida creciente en la última década. Sin embargo, la mayor parte de la producción científica carece de marcos conceptuales y debates académicos. Por otra parte, se advirtió que el empresariado ha sido un tema ampliamente abordado desde las ciencias económicas y con menor intensidad por la sociología. El enfoque de la economía analiza a las organizaciones con un determinismo estructural en función del crecimiento económico. Situación similar acontece en la sociología la cual privilegia los elementos estructurales y objetivos.

Este artículo mostró que los referentes teóricos empleados tradicionalmente por los enfoques de la economía y la sociología han sido insuficientes para comprender el fenómeno del empresariado, puesto que las organizaciones son productos de la acción social y por lo tanto no tiene sentido estudiarlas sin la inclusión del hombre. Con el fin de superar la situación descrita, se propuso la inclusión de la teoría de la acción social desde la perspectiva construida por Pierre Bourdieu, como una alternativa que oriente la historiografía y los debates en torno al empresario colombiano.

Bajo los preceptos de Bourdieu, el empresario es un agente que se encuentra inmerso e interactúa en un sistema de relaciones. De esta manera, si pretendemos atender la realidad específica y dar cuenta de la manera como surgió el conjunto de organizaciones que conforman la actividad acuícola en el departamento del Huila, es imprescindible abordar el estudio del empresario, en la concepción del agente que produce realidades a través sus prácticas y que llegó a conformar un campo empresarial.

El estudio de los empresarios de la actividad acuícola conlleva a identificar las representaciones de sentido que él se construye, así como la manera en que se procuró los beneficios. Es decir, abordar las prácticas, tales como las redes de relaciones sociales que teje, los vínculos que forma con la esfera pública para el uso de los recursos y de estas instancias, su inclusión en otros campos, las alianzas con agentes de su mismo campo y la manera como da forma a su reconocimiento, entre otros. Como agentes, estos empresarios estuvieron y/o están en capacidad de maniobrar, por lo que procuran la incorporación y reproducción de capital, con el cual se hacen partícipes y da forma a un campo. Desde esta óptica, el campo empresarial acuícola es el espacio de acción de unos agentes que ponen en juego y luchan por un capital, no sólo material, sino simbólico.

La organización empresarial es un resultado objetivo del juego de unos actores en un campo. Cualquier intento de comprenderlas alejadas de sus productores, es decir asumirlas como objetos acabados, constituye algo azaroso e incompleto.

\section{Referencias}

Beltrán, C. y Villaneda, A. (2000). Perfil de la pesca y la acuicultura en Colombia. Bogotá: Instituto Nacional de Pesca y Acuicultura.

Becattini, G. (1988). Los distritos industriales y el reciente desarrollo italiano. En: Distritos industriales y pequeña empresa. España: Siglo Veintiuno.

Bourdieu, P. (2011). Las estrategias de la reproducción social. Buenos Aires: Siglo Veintiuno.

Bourdieu, P. (2012). Bosquejo de una teoría de la práctica. Buenos Aires: Prometeo Libros. 
Bourdieu, P. (1988). La distinción. Crítica y bases sociales del gusto. Madrid: Tauros.

Castillo L.F. (2000). La importancia de la tilapia roja en el desarrollo de la piscicultura en Colombia. Cali: Asociación Red Cauca, Alevinos del Valle.

Castillo, L. F. (2003). Tilapia Roja: una evolución de 20 años, de la incertidumbre al éxito. Cali: Asociación Red Cauca, Alevinos del Valle.

Cerruti, M. (2004). Los estudios empresariales en América Latina. ¿El debate interminable?. En: Las regiones y la historia empresarial. Medellín: Universidad Eafit.

Chia, R. y MacKay, B. (2007). Post-processual challenges for the emerging strategy-aspractice perspective: Discovering strategy in the logic of practice. Human Relations; 60; 217. Sage.

Dávila, C. (1986). El empresario colombiano, una perspectiva histórica. Bogotá: Pontificia Universidad Javeriana.

Dávila, C. (1996). Historia empresarial deColombia: estudios, problemas y perspectivas. Bogotá: Universidad de los Andes.

Dávila, C. (1996). Empresas e historia en América Latina. Un balance historiográfico. Bogotá: TM Editores - Colciencias.

Dávila, C. (2003). Empresa y empresarios en la historia de Colombia. Siglos XIX y XX. Bogotá: Grupo Editorial Norma.

Dimaggio, P. y Powell, W. (1999). El nuevo institucionalismo en el análisis organizacional. México, D.F.: Fondo de Cultura Económica.

Gobernación del Huila. (2013). Informe de cadena piscícola Huila. Extraído el 01 de septiembre de 2013 de http://www.huila. gov.co/documentos
Gómez M. (2005). Competitividad y complejos productivos: teoría y lecciones de política. Estudios y perspectivas número 27. Buenos Aires: Cepal, Naciones Unidas.

Goodin, R. (2003). Las instituciones y su diseño, en: Teoría del diseño institucional, Goodin Robert E. España: Gedisa.

Gutiérrez, A. (2011). Clases, espacio social y estrategias, una introducción al análisis social en Bourdieu. En: P. Bourdieu, La estrategia de la reproducción social (pp. 9-27). Buenos Aires: Siglo Veintiuno.

Humprhrey, J. (1995). Industrial reorganization in developing countries: from models to trajectories. Wordl Development, Vol. 23, No. 1, 149-162.

Inpa. (1997). Boletín estadístico. Bogotá: Instituto Nacional de Pesca.

Jepperson, R. (1991). Instituciones, efectos institucionales y la institucionalidad. En: The New Institutionalism in Organizational Analysis, Paul J. Dimaggio y Walter W. Powell. Chicago: University of Chicago Press. págs. 143-163.

López, L. y Calderón, G. (2006). Análisis de las dinámicas culturales al interior de un clúster empresarial. Estudios Gerenciales, abril-junio, 13-37.

March,J.G.yOlsen,J.P.(1997)Elredescubrimiento de las instituciones. La base organizativa de la política. México: Fondo de Cultura Económica, Colegio Nacional de Ciencias Políticas y administración Pública, A. C., Universidad Autónoma de Sinaloa.

Meyer, John W. y Brian Rowan (1977) "Institutionalized Organizations: Formal structure as myth and ceremony". En: The American Journal of Sociology, vol. 83, Núm. 2, págs. 340-363.

Piña, J. y Morales, A. (2010). Aloe en Venezuela: de la cadena de valor al Distrito Industrial. 
Problemas del desarrollo. Revista Latinoamericana de Economía, 41(160), 187-208.

Porter, M. (1998). Agrupaciones y la nueva economía de la competencia. Harvard Business Review, 76 (6), 77-90.

Rosenfeld, Stuart (2002), “Creating Smart Systems: A Guide to clusters strategies in less favored regions", Regional Technology Strategies, Carboro, North Carolina, USA. Extraído el 07 de septiembre de www. rtsinc.org
Scapens, R. W. (1994). Never mind the gap: towards an institutional perspective on management accounting practice. Management Accounting Research 5, 301-321.

Schmitz, H. (1995), “Collective Efficiency: Growth path for small-scale industry. The Journal of development studies, vol. 31, No. 4, p. $529-566$.

Scott, W. Richard (2008), Institutions and Organizations, Sage, California, pp. 47-71. 